Canadian

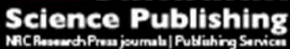

Applied Physiology, Nutrition, and Metabolism Physiologie appliquée, nutrition et métabolisme

\title{
A single session of exhaustive exercise markedly decreases circulating levels of guanidinoacetic acid in healthy men and women
}

\begin{tabular}{|r|l|}
\hline Journal: & Applied Physiology, Nutrition, and Metabolism \\
\hline Manuscript ID & apnm-2016-0102.R3 \\
\hline Manuscript Type: & Brief communication \\
\hline Date Submitted by the Author: & 13-Jun-2016 \\
\hline & $\begin{array}{l}\text { Stajer, Valdemar; Univerzitet u Novom Sadu } \\
\text { Trivic, Tatjana; Univerzitet u Novom Sadu } \\
\text { Drid, Patrik; Univerzitet u Novom Sadu } \\
\text { Vranes, Milan; Univerzitet u Novom Sadu } \\
\text { Ostojic, Sergej; University of Novi Sad, Biomedical Sciences }\end{array}$ \\
\hline Keyword: & Bioenergetics, Creatine, Creatinine, Running-to-Exhaustion \\
\hline
\end{tabular}


BRIEF COMMUNICATION

\title{
A single session of exhaustive exercise markedly decreases circulating levels of guanidinoacetic acid in healthy men and women
}

\author{
Valdemar Stajer ${ }^{1}$, Tatjana Trivic ${ }^{1}$, Patrik Drid ${ }^{1}$, Milan Vranes ${ }^{2}$, Sergej M. Ostojic ${ }^{1,3^{*}}$ \\ ${ }^{1}$ Faculty of Sport and Physical Education, University of Novi Sad, Serbia \\ ${ }^{2}$ Faculty of Sciences, University of Novi Sad, Serbia \\ ${ }^{3}$ University of Belgrade School of Medicine, Belgrade, Serbia
}

\begin{tabular}{lc} 
Word count & 2860 \\
\hline No. of figures & 1 \\
\hline No. of references & 17
\end{tabular}

\section{Correspondence:}

Prof. Sergej M. Ostojic, MD, PhD, Applied Bioenergetics Lab, Biomedical Sciences Department, Faculty of Sport and Physical Education, University of Novi Sad, Lovcenska 16, Novi Sad 21000, Serbia. Tel.: (++381)-21-450-188; Fax: (++381)-21-450-199; E-mail: sergej.ostojic@,chess.edu.rs 


\begin{abstract}
We evaluated the effects of exercise on circulating concentrations of guanidinoacetic acid (GAA) and creatine in 23 healthy volunteers subjected to running to exhaustion and free-weight bench-press to volitional failure. Blood was taken before and following each exercise session. Running induced a significant decrease in serum GAA by $20.1 \%$ $(P<0.001)$, while free-weight exercise reduced GAA by $11.7 \%(P<0.001)$, suggesting the possible use of serum GAA as a novel biomarker of exhaustion.
\end{abstract}

Keywords Bioenergetics; Creatine; Creatinine; Running-to-Exhaustion; Bench press 


\section{Introduction}

Guanidinoacetic acid (GAA) is a naturally-occurring amino acid derivative of glycine, and the metabolic forerunner of creatine, a compound that helps to supply energy to all cells, including the skeletal muscle, brain and heart (Wallimann et al. 2011). GAA is metabolized to creatine in a reaction catalyzed by guanidinoacetate $N$ methyltransferase (GAMT), with creatine synthesis mainly occuring in the liver, pancreas and kidneys (Brosnan et al. 2011). Although GAA has been identified as an endogenous substance in humans a long time ago (Hoberman 1947), and a recent study recognized its specific effect on brain cell development (Hanna-El-Daher et al. 2015), GAA metabolism is rather poorly described in the scientific literature, particularly during different bioenergetic challenges. This is quite surprising compared to its derivative creatine, which is well described in terms of utilization during energy-demanding circumstances, such as exercise or tissue injury (Sotgia et al. 2007; Lindbom et al. 2008; Lygate et al. 2013). In particular, no study known to the authors evaluated serum GAA utilization during heavy exercise in humans. Since exhaustive exercise markedly alters kidney function (Bellinghieri et al. 2008), with the kidney being a major organ for GAA production (Brosnan et al. 2011), monitoring GAA levels during exercise might be a useful biomarker of heavy exercise-induced kidney stress. A possible exercise-induced GAA depletion may result in disrupted creatine production, and jeopardize transfer and utilization of cellular energy that consequently could compromise exercise performance that depends on a high turnover of phosphocreatine (such as high-intensity short duration activities or the beginning/end of long duration exhaustive exercise). Alternatively, GAA depletion might be caused by increased creatine synthesis rate during exercise. Therefore, the main aim of this observational study was to evaluate the effects of a single exhaustive exercise session on circulating concentrations of GAA, creatine and creatinine in healthy men and women.

\section{Methods}

\section{Participants}

Twenty-three young apparently healthy volunteers (12 men and 11 women) gave informed consent to participate in this study. Sample size $(n=23)$ was calculated using the power analysis (one-tailed, effect size $0.5, \alpha 0.05$, power 0.80) for the primary outcome measure, exercise-induced drop in serum GAA concentrations (G-Power 3, Heinrich Heine University Düsseldorf, Germany), with the effect size previously categorized as medium in a related study (Ostojic et al. 2013). The mean physical characteristics of participants were: age $24.7 \pm 2.5$ years, height $174.9 \pm$ 
$10.0 \mathrm{~cm}$, and weight $69.6 \pm 13.2 \mathrm{~kg}$. All participants were free from cardio-metabolic diseases and musculoskeletal disorders, moderately physically active ( $\sim 5$ hours per week), and were not taking any medication or dietary supplementation. The study was conducted in accordance with the Declaration of Helsinki, with study approved by the local IRB at the University of Novi Sad Faculty of Sport and Physical Education (Ref. ID 031/15-ASN:228).

\section{Study design}

All volunteers were assessed on two occasions separated by 7 days, with participants subjected to a single session of exhaustive exercise at each visit to the lab. Measurements were carried out between 08:00 and 11:00 after an overnight fast, and no heavy exercise over the previous $24 \mathrm{~h}$. During the first visit, participants were assigned to incremental running-to-exhaustion test on an institutional treadmill (3-min warm-up walk at $6 \mathrm{~km} / \mathrm{h}$ followed by running at $8 \mathrm{~km} / \mathrm{h}$ with progressive workload increment rate of $1.5 \mathrm{~km} / \mathrm{h}$ every $60 \mathrm{sec}$ until exhaustion). Test was finished when participants were physical tired to continue running, with subjective rates of perceived exertion (RPE; Buckley and Borg 2011) and heart rates monitored during the test (Polar S 810, Oy Kempele, Finland). At second visit to the lab, participants performed free-weight bench press exhaustive exercise (5-repetition warm-up with $10 \mathrm{~kg}$ followed by bench press repetitions at workload equal to $25 \%$ of participants' weight until volitional failure). All participants were familiar to testing procedures as part of their training routine. Two different modes of exhaustive exercise were chosen due to possible mode-response interaction with renal function and metabolism, with heavy aerobic exercise particularly effective to compromise kidney function as compared to resistance exercise (Bellinghieri et al. 2008; Moinuddin and Leehey 2008).

\section{Clinical chemistry}

At each lab session, blood samples were drawn from an antecubital vein into a gel vacutainer before (fasting) and immediately after exercise. Gel vacutainers were immediately centrifuged at $3000 \mathrm{~g}$ for $15 \mathrm{~min}$, with serum stored at $-20^{\circ} \mathrm{C}$ and analyzed for GAA and creatine after the completion of the study using modified LC-MS/MS (Agilent 1200 Series LC System, Agilent Technologies Inc., Santa Clara, CA, USA). The separation of the analytes was achieved on a Kinetex ${ }^{\circledR H I L I C ~ c o l u m n ~}(100 \mathrm{~mm} \times 2.1 \mathrm{~mm}$ i.d., $2.6 \mu \mathrm{m})$ (Phenomenex Inc., Torrance, CA, USA) with a column temperature of $30^{\circ} \mathrm{C}$. The mobile phase consisted of three eluents, methanol $(10 \%)$, acetonitrile $(80 \%)$ and ammonium formate $(\mathrm{pH} 3.7,10 \%)$ delivered with isocratic elution at a flow rate of $0.5 \mathrm{~mL} / \mathrm{min}$. 
Creatinine was analyzed by standard colorimetric method (Heinegård and Tiderström 1973).

\section{Statistical analyses}

When homogenous variances were verified for normally distributed data, baseline vs. follow-up measures were compared by two-way mixed model ANOVA with repeated measures to establish if any significant differences existed between participants' responses over time (baseline vs. post-exercise), with exercise mode (running to exhaustion or free-weight bench-press) included as a between-subjects factor. When non-homogenous variances were identified, values were compared using Kruskal-Wallis test. The relationship between changes in serum GAA and creatine levels was examined using Pearson's product-moment correlation coefficient. Significance level was set at $P \leq 0.05$. The data were analyzed using the statistical package SPSS, PC program, version 21.0 (SPSS Inc., Chicago, Illinois, USA).

\section{Results}

Participants successfully completed both sessions of exhaustive exercise, with running test ended in $371 \pm 94 \mathrm{~s}$, with end-exercise RPEs $9.9 \pm 0.2$ and peak heart rate $189 \pm 8$ beats per min $(98.5 \pm 3.7 \%$ of age-predicted maximal heart rate), while bench press test was finished after $57 \pm 34$ repetitions (test ended in $199 \pm 86$ s), and end-exercise RPEs $9.9 \pm 0.2$. Baseline levels of fasting serum biomarkers were: GAA $3.0 \pm 0.8 \mu \mathrm{mol} / \mathrm{L}$, creatine $22.5 \pm 8.0 \mu \mathrm{mol} / \mathrm{L}$, and creatinine $6.9 \pm 2.0 \mathrm{mg} / \mathrm{L}$ before running-to-exhaustion test; and GAA $2.6 \pm 0.8 \mu \mathrm{mol} / \mathrm{L}$, creatine $20.6 \pm 6.4 \mu \mathrm{mol} / \mathrm{L}$, and creatinine $7.6 \pm 1.0 \mathrm{mg} / \mathrm{L}$ before free-weight bench press test. We found that exercise markedly affected circulating GAA, with both modes of exhaustive exercise induced a significant drop in serum GAA $(2.4 \pm 0.7$ $\mu \mathrm{mol} / \mathrm{L}$ after running test, and $2.3 \pm 0.8 \mu \mathrm{mol} / \mathrm{L}$ after bench press test) compared to the baseline concentrations $(P<$ 0.05). The effects are markedly superior after incremental running-to-exhaustion test comparing to free-weight bench press to volitional failure $(P=0.02$; Figure 1$)$. The highest alteration was found in a female participant who experienced a drop in serum GAA from $4.26 \mu \mathrm{mol} / \mathrm{L}$ (at baseline) to $2.14 \mu \mathrm{mol} / \mathrm{L}$ (post-exercise) after running-toexhaustion test. The least reduction in serum GAA during the study $(0.7 \%)$ was reported in a man after free-weight bench press test, and four participants (one women after running-to-exhaustion test, and two women and a men after bench press test) experienced a rise in serum GAA (from 1.0\% to $14.9 \%$ ) post-exercise. In addition, serum creatine was elevated after running-to-exhaustion exercise by $3.2 \mu \mathrm{mol} / \mathrm{L}(95 \% \mathrm{CI}=3.0-3.4 \mu \mathrm{mol} / \mathrm{L} ; P=0.004)$, while 
free-weight exercise had no apparent effect on serum creatine (post-exercise levels were $23.9 \pm 6.4 \mu \mathrm{mol} / \mathrm{L} ; P=$ 0.07), and no differences were found in creatine upsurge from baseline to follow-up between two modes of exhaustive exercise applied $(P=0.22)$. Finally, both modes of exhaustive exercise significantly elevated serum creatinine comparing to baseline levels $(9.7 \pm 1.2 \mathrm{mg} / \mathrm{L}$ after running test, and $7.4 \pm 1.4 \mathrm{mg} / \mathrm{L}$ after bench press test; $P<0.05$ ), with running-to-exhaustion found to be more powerful to elevate serum creatinine as compared to bench press exercise $(P<0.001)$. A moderate-to-strong negative linear correlation was reported between post-exercise changes in GAA and creatine for both exhaustive running $(r=-0.67 ; P<0.001)$ and free-weight exercise $(r=-0.39$; $P=0.03)$ for one-tailed probabilities, respectively.

- Figure 1 about here -

\section{Discussion}

The present study showed a notable drop in serum GAA concentration in response to a single session of exhaustive exercise, with lowest values occurring immediately after endurance running exercise in healthy men and women. It seems that longer-duration exhaustive exercise that recruits large muscle groups (both legs and arms) generates a greater reduction in GAA availability, suggesting a mode-response interaction between an exhaustive workout and GAA utilization. Furthermore, serum creatine and creatinine are sensitive to different modes of exhaustive exercise.

GAA (also known as guanidinoacetate, glycocyamine or $N$-amidinoglycine) is a natural metabolite of glycine (aminoacetic acid) in which the amino group has been converted into a guanidine. The metabolic role of GAA in cellular bioenergetics, as a natural precursor of creatine and an alternative substrate for creatine kinase, has been well described (Ostojic 2015). After synthesized from glycine and $L$-arginine in the kidney and pancreas, GAA circulates to the liver (also kidney, pancreas, and reproductive organs) to yield creatine through the essential methylation reaction catalyzed by GAMT, using $S$-adenosyl methionine as the methyl donor (Brosnan et al. 2011). Normal endogenous creatine production from GAA in humans accounts for $\sim 1$ gram per day, with additional creatine provided by diet (about $1 \mathrm{~g} /$ day, mainly from meat) serves to maintain normal creatine homeostasis (Wyss and Kaddurah-Daouk 2000). Several clinical studies reported different disorders in endogenous GAA and creatine production, leading to severe impairments of cellular bioenergetics and clinical manifestations (Schulze 2013), however limited information is available regarding GAA physiology during energy-demanding circumstances in 
healthy humans. In this study, we found an exhaustive exercise-induced diminution of GAA availability, as assessed by a notable acute drop in serum GAA post-exercise. Consequently, heavy exercise could be considered as a GAAdepleting intervention; this might be due to an exercise-induced reduction in GAA production, an increase in GAA utilization to creatine, or both. Since heavy exercise induces complex changes in the renal hemodynamics including a reduction in blood flow and metabolism (Bellinghieri et al. 2008), this might have an acute negative effect on GAA synthesis in the main GAA-producing organ by reducing the availability of glycine and $L$-arginine, or by suppressing the activity of $L$-arginine:glycine amidinotransferase, GAA synthesizing enzyme. Previous study by Japanese group reported low serum GAA in patients suffering from chronic kidney disease or diabetes mellitus (Tsubakihara et al. 2012), suggesting possible link between kidney dysfunction and GAA deficiency. Alternately, a bout of heavy exercise might acutely stimulate GAA methylation to creatine (and creatine to creatinine) which causes GAA depletion and creatine boosting, particularly during brief exercise where creatine is considered a main source of cellular energy. Here, we reported increased serum creatine levels after exercise, implying enhanced GAA utilization to creatine by exhaustive exercise, perhaps driven by enhanced GAMT activity. Further studies with isotopic GAA tracer along with enzyme activity/expression assays are needed to evaluate the contribution of each factor for GAA utilization during energy-demanding circumstances such as exercise. In addition, gender-related differences in GAA dynamics following exercise need to be addressed in future studies, since men and women seem to differ in creatine-related markers after high-intensity exercise (Willcocks et al. 2010). This was not addressed during the present trial due to small subsamples of men and women. Furthermore, we compared does two different modes of exhaustive exercise had a different impact on GAA utilization in healthy men and women. It seems that endurance running induced a more pronounced fall in serum GAA immediately post-exercise as compared to weight lifting (21.3 vs. 11.2\%). This might illustrate a mode-response effect of heavy exercise on GAA availability, considering the fact that endurance running-to-exhaustion was of longer duration and engaged more skeletal muscle (a major organ of creatine utilization) that probably consumes and/or utilizes more GAA. Alternatively, heavy aerobic exercise might induce profound changes in the renal hemodynamics and metabolism that consequently reduces GAA synthesis in the kidney and its availability in the circulation. This hypothesis should be further evaluated since the current study did not test any other markers of kidney damage, and perhaps validate serum GAA as a biomarker of exercise-induced kidney stress. On the other hand, exhaustive resistance exercise seems to less aggressively affect GAA-creatine cycle, inducing rather moderate decrease in serum GAA while serum creatine 
tended to increase at post-exercise. This might be due to either lower bioenergetic cost or minor kidney dysfunction imposed by this mode of exercise, as compared to aerobic exercise.

In conclusion, circulatory GAA seems to be responsive to acute exhaustive exercise, with serum GAA availability depleted from $3.4 \%$ up to $49.8 \%$ (maximum change) by extended and all-encompassing exercise. GAA should be further evaluated as a possible biomarker of exercise load, with additional studies needed to describe physiological background and possible consequences of exercise-induce GAA depletion.

\section{Conflict of interest}

The authors declare that there are no conflicts of interest.

\section{Acknowledgements}

This study was supported by the grants from the Serbian Ministry of Education, Science and Technological Development (Grant No. 175037), the Provincial Secretariat for Science and Technological Development (grant number 114-451-710/2016-03), and the Faculty of Sport and Physical Education, University of Novi Sad (2015 Annual Award).

\section{References}

Bellinghieri G, Savica V, Santoro D. 2008. Renal alterations during exercise. J. Ren. Nutr. 18: 158-164. doi: 10.1053/j.jrn.2007.10.031. PMID: 18089464.

Buckley JP, Borg GA. 2011. Borg's scales in strength training; from theory to practice in young and older adults. Appl. Physiol. Nutr. Metab. 36: 682-692. doi: 10.1139/h11-078. PMID: 21977913.

Brosnan JT, da Silva RP, Brosnan ME. 2011. The metabolic burden of creatine synthesis. Amino Acids. 40: 13251331. doi: 10.1007/s00726-011-0853-y. PMID: 21387089.

Hanna-El-Daher L, Béard E, Henry H, Tenenbaum L, Braissant O. 2015. Mild guanidinoacetate increase under partial guanidinoacetate methyltransferase deficiency strongly affects brain cell development. Neurobiol. Dis. 79: 14-27. doi: 10.1016/j.nbd.2015.03.029. PMID: 25896543.

Heinegård D, Tiderström G. 1973. Determination of serum creatinine by a direct colorimetric method. Clin. Chim. Acta. 43: 305-310. doi:10.1016/0009-8981(73)90466-X. PMID: 4690902 
Hoberman HD. 1947. The determination of guanidoacetic acid and arginine in human urine and serum. J. Biol. Chem. 167: 721-727. PMID: 20287903.

Lindbom M, Ramunddal T, Camejo G, Waagstein F, Omerovic E. 2008. In vivo effects of myocardial creatine depletion on left ventricular function morphology and lipid metabolism: study in a mouse model. J. Card. Fail. 14: 161-166. doi: 10.1016/j.cardfail.2007.10.020. PMID: 18325464.

Lygate CA, Schneider JE, Neubauer S. 2013. Investigating cardiac energetics in heart failure. Exp. Physiol. 98: 601605. doi: 10.1113/expphysiol.2012.064709. PMID: 22983996.

Moinuddin I, Leehey DJ. 2008. A comparison of aerobic exercise and resistance training in patients with and without chronic kidney disease. Adv. Chronic Kidney Dis. 15: 83-96. doi: 10.1053/j.ackd.2007.10.004. PMID: 18155113

Ostojic SM. 2015. Cellular bioenergetics of guanidinoacetic acid: the role of mitochondria. J. Bioenerg. Biomembr. 47: 369-372. doi: 10.1007/s10863-015-9619-7. PMID: 26255041.

Ostojic SM, Niess B, Stojanovic M, Obrenovic M. 2013. Creatine metabolism and safety profiles after six-week oral guanidinoacetic acid administration in healthy humans. Int. J. Med. Sci. 10: 141-147. doi: 10.7150/ijms.5125. PMID: 23329885

Schulze A. 2013. Creatine deficiency syndromes. Handb. Clin. Neurol. 113: 1837-1843. doi: 10.1016/B978-0-44459565-2.00053-8. PMID: 23622406.

Sotgia S, Carru C, Caria MA, Tadolini B, Deiana L, Zinellu A. 2007. Acute variations in homocysteine levels are related to creatine changes induced by physical activity. Clin. Nutr. 26: 444-449. doi: 10.1016/j.clnu.2007.05.003. PMID: 17582661.

Tsubakihara Y, Terumasa H, Tatsuya S. 2012. Guanidinoacetic acid (GAA) in patients with chronic kidney disease (CKD) and diabetes mellitus (DM). Kid. Res. Clin. Pract. 31: A81. doi:10.1016/j.krcp.2012.04.581.

Wallimann T, Tokarska-Schlattner M, Schlattner U. 2011. The creatine kinase system and pleiotropic effects of creatine. Amino Acids. 40: 1271-1296. doi: 10.1007/s00726-011-0877-3. PMID: 21448658.

Willcocks RJ, Williams CA, Barker AR, Fulford J, Armstrong N. 2010. Age- and sex-related differences in muscle phosphocreatine and oxygenation kinetics during high-intensity exercise in adolescents and adults. NMR Biomed. 23: 569-577. doi: 10.1002/nbm.1495. PMID: 20661873. 
Wyss M, Kaddurah-Daouk R. 2000. Creatine and creatinine metabolism. Physiol Rev. 80: 1107-1213. PMID: 10893433. 


\section{Figure caption}

Figure 1. Changes from baseline (\%) for serum GAA, creatine and creatinine levels at post-exercise. Values are presented as means, with upper and lower bounds represent $95 \%$ confidence intervals. * Indicates significant difference baseline vs. post-exercise $(P<0.05) . \dagger$ Indicates significant difference between running-to-exhaustion and free-weight bench press exercise $(P<0.05)$. 


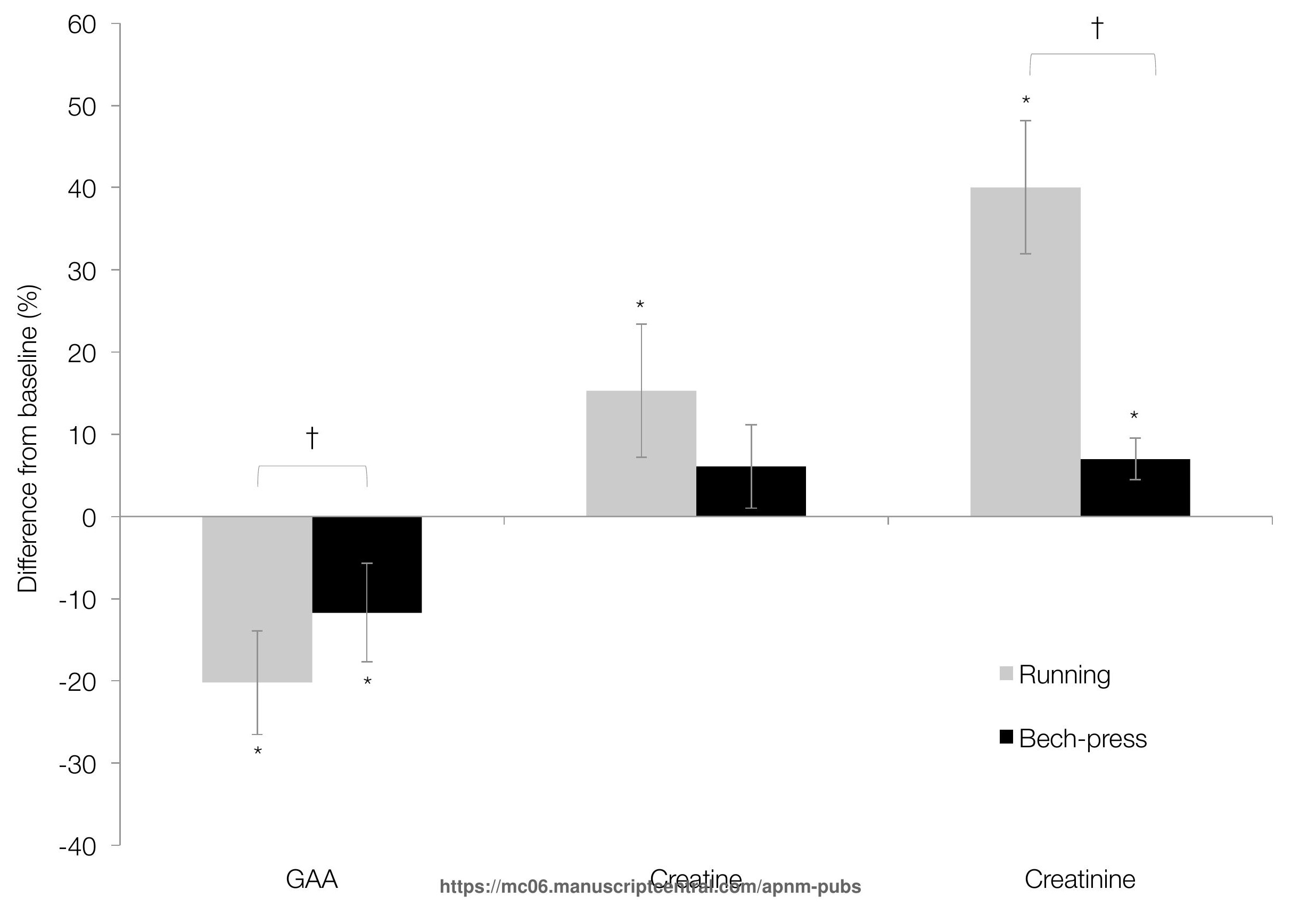

